\title{
Multi-wavelength compressive computational ghost imaging
}

\author{
Stephen S. Welsh ${ }^{a}$, Matthew P. Edgar ${ }^{a}$, Phillip Jonathan ${ }^{b}$, Baoqing Sun ${ }^{a}$, Miles. J. Padgett ${ }^{a}$ \\ ${ }^{a}$ University of Glasgow, Kelvin Building University Avenue G12 8QQ, Glasgow, UK; \\ ${ }^{b}$ Department of Mathematics and Statistics, Lancaster University, Lancaster, LA1 4YF, UK;
}

\begin{abstract}
The field of ghost imaging encompasses systems which can retrieve the spatial information of an object through correlated measurements of a projected light field, having spatial resolution, and the associated reflected or transmitted light intensity measured by a photodetector. By employing a digital light projector in a computational ghost imaging system with multiple spectrally filtered photodetectors we obtain high-quality multi-wavelength reconstructions of real macroscopic objects. We compare different reconstruction algorithms and reveal the use of compressive sensing techniques for achieving sub-Nyquist performance. Furthermore, we demonstrate the use of this technology in non-visible and fluorescence imaging applications.
\end{abstract}

Keywords: Ghost Imaging, Single Pixel Detectors, DLP Technologies, Multi-wavelength Imaging, Non-visible Imaging, Fluorescence Imaging

\section{INTRODUCTION}

Ghost imaging (GI) has been an active research area for nearly two decades. First demonstrated utilizing spatially entangled photons, ${ }^{1}$ it was later shown possible using classical correlations of pseudo-thermal light sources. ${ }^{2-5}$ Early demonstrations of so called 'classical GI' used a laser beam propagated through a ground glass diffuser in order to produce a pseudo-thermal speckle field. A beam splitter was used to produce two identical copies of the field which were subsequently propagated along two different paths: the test path and the reference path. The test path contained a partially transmissive or reflective object (typically planar) and a single-pixel photodetector (SPD) with no spatial resolution, whereas the reference path contained a detector with spatial resolution, typically a charged coupled device (CCD). Although the object was never seen by a detector with spatial resolution, it was by correlating the measurements of the reference and test paths that one can reconstruct an estimate of the object which improves after every iteration. However, recent advances in the field, ${ }^{7}$ such as the use of programmable light fields, via spatial light modulators (SLM), has removed the need for the beam splitter and the CCD array, thereby reducing the number of components necessary for GI systems: a technique called computational GI. ${ }^{6}$ Furthermore, improvements in computer technology, such as greater RAM capacity, have enabled the prospect of single-element imaging devices for advanced imaging applications, which do not rely on a raster scanning techniques.

\section{EXPERIMENTAL SETUP}

In this experiment we adapt a computational GI setup, as shown in Figure 1, by replacing the SLM and laser with a digital light projector (DLP) (Texas Instruments LightCommander). The DLP contains a digital micro-mirror device (DMD) and four light emitting diodes: red (R), green $(\mathrm{G})$, blue (B) and near-infrared (NIR), which can produce structured digital light fields. We utilise four different spectrally filtered single-pixel photodetectors to measure the intensity of the reflected light at each of the emitted wavelengths, an analogue to digital converter to digitize the photodetector signals and a computer to generate the random illumination patterns and perform multi-wavelength reconstructions of the test object. In this demonstration we utilise the built-in illumination sources of the DLP with frequencies in the extended visible band $(400 \mathrm{~nm}-850 \mathrm{~nm})$, however the large operational bandwidth of the DMD $(300 \mathrm{~nm}-2 \mu \mathrm{m})$ enables the use of this technique at wavelengths that are potentially unsuitable for existing imaging technologies.

(Send correspondence to Stephen S.Welsh)

Stephen S.Welsh: E-mail: s.1.welsh@research.gla.ac.uk, Telephone: 07748080308 


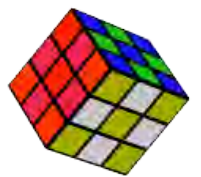

object

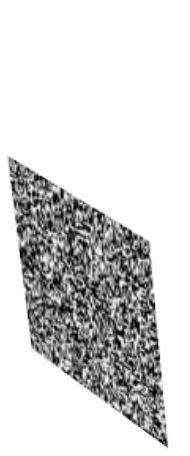

illumination pattern digital light

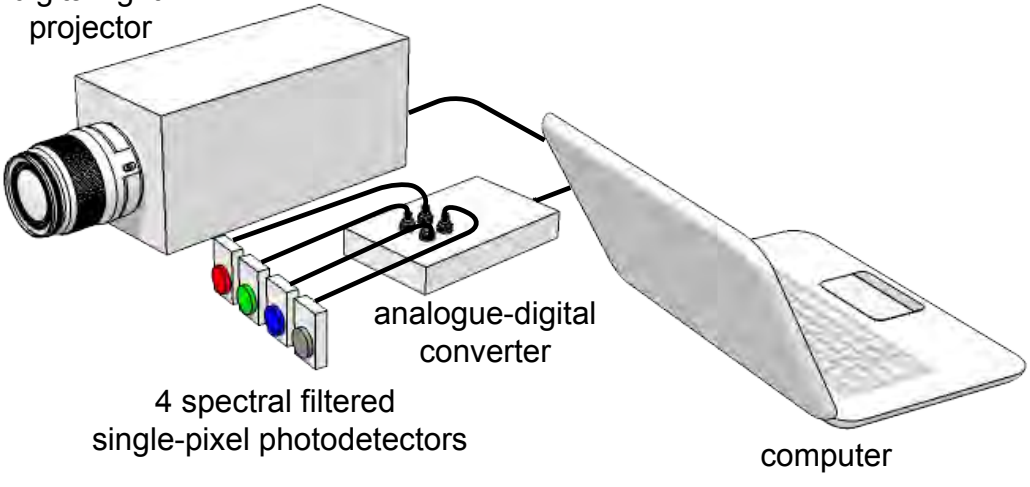

Figure 1. 3D schematic of the experimental system where the Rubik's Cube test object had selected sections painted in fluorescent ink. The LED illumination chart describes the two main illumination modes which the projector can be set in. In video mode, the internal LED illuminate in sequence as shown with IR unavailable, conversely all LED's remain in simultaneous emission in structured light mode.

The patterns we use are randomly distributed binary patterns, having a fixed black and white ratio $1: 1$ which provides self-normalisation in intensity output of the system. These fixed ratio patterns are then projected onto the object using a Nikon 50mm lens. Four spatially separated single-pixel photodetectors are positioned to monitor the back-reflected light. For every pattern projected, the corresponding object intensity is measured by each photodetector, which is fed to a computer algorithm.

Normally during the data acquisition process, all external sources of light must be eliminated from the system since they would interfere with the recorded voltage from the SPD's, this requires ghost imaging to be done in the dark, however in this system we take advantage of the 24 bit-planes projected by the DLP, normally used for color depth, to overcome such external noise sources. By alternating between a binary pattern and its inverse in subsequent bit planes we can demodulate the measured signal at the frequency of the bit plane projection to isolate the back-reflected signal from light sources at other frequencies. Importantly, the use of a unity black and white ratio pattern enables normalisation of the measured signals for each pattern which has been shown to improve the SNR of the final reconstruction. ${ }^{8,9}$

Several approaches can be undertaken to produce multi-wavelength ghost images. One approach is to perform time sequenced illumination of different wavelengths with an unfiltered SPD synchronised with the changes in the illumination sequence. Alternatively this could also be achieved by simultaneous broadband illumination from all LED's with multiple SPD's filtered for different frequencies. The approach that is described in this work is a hybrid of these two approaches, namely time sequenced illumination with multiple filtered SPD's. Using any of the aforementioned techniques, each separate ghost image retrieved indicates a different color plane according to the spectral content of the illumination or the filter used. The reconstruction quality is determined by the algorithm which is implemented, for which two main groups exist; iterative and inversion.

\section{GHOST IMAGING ALGORITHMS}

\subsection{Traditional ghost imaging}

Iterative algorithms make a refined estimate reconstruction with a resolution, $N$ of the object after a number of measurements $M$, have been made. For each iteration a unique 2D pattern $I(x, y)$ is projected and voltage signal from a filtered $\mathrm{SPD}, S_{\mu}$, are acquired and can be expressed as

$$
S_{\mu}=\int I(x, y) R_{\mu}(x, y) d x d y,
$$

where $R_{\mu}(x, y)$ is the object reflection function for a spectral frequency $\mu$. Thus the overlap of the pattern and the object determines the measured signal and hence weighting factor. From the correlation of known patterns 
and reflected signals it follows that

$$
O_{\mu}(x, y)=<S_{\mu} I(x, y)>-<S_{\mu}><I(x, y)>,
$$

where $O_{\mu}(x, y)$ is our object reconstruction and $\langle\ldots\rangle$ denotes an ensemble average of these values, hence this is an iterative process. Equation 2 has been the basis for much of the earlier work in GI and is referred to herein as traditional GI (TGI). Over many iterations, on the order of $M \approx N$, an image of the object is reconstructed. This highlights some impracticalities of TGI, where a traditional raster scan process could match the base performance of this approach.

\subsection{Compressive sensing}

Inversion algorithms use all the data in a bulk process to find the best solution for a set of unknowns, which in this case is the object. To do this requires reshaping each pattern, $I_{m}$, as a $1 \mathrm{D}$ array and generating a large pattern matrix for all iterations, $M$, such that

$$
\mathbf{I}=\left[\begin{array}{llll}
I_{1} & I_{2} & \ldots & I_{M}
\end{array}\right]^{T},
$$

and the measured signals are vectorized such that

$$
\mathbf{S}_{\mu}=\left[\begin{array}{llll}
S_{\mu 1} & S_{\mu 2} & \ldots & S_{\mu M}
\end{array}\right]^{T},
$$

From this point the problem becomes one of matrix inversion where the $M \times N$ pattern matrix $\mathbf{I}$ is matched with the measured signal vector $\mathbf{S}_{\mu}$. With such an approach if $M=N$ (the Nyquist limit), this can be solved by least-squares methods. However, since the number of measurements required scales directly with the number of pixels in the image, the size of $\mathbf{I}$ makes the reconstruction computationally intensive at higher resolutions. Using fewer measurements than the number of pixels (ill-conditioned problem) the image quality is rapidly reduced.

Compressive ghost imaging ${ }^{10,11}$ (CGI) is an alternative inverse approach that can maintains image quality with ill-conditioned (sub-Nyquist) systems by taking advantage of the natural sparsity in images. In the method that is used here we first perform a $2 \mathrm{D}$ discrete cosine transform (DCT) on the pattern matrix such that $\mathbf{I} \Rightarrow \mathbf{I}_{D C T}$. The ill-conditioned problem can then be expressed as

$$
\mathbf{I}_{D C T} \cdot \mathbf{O}_{\mu}^{\prime}=\mathbf{S}_{\mu}
$$

where $\mathbf{O}^{\prime}{ }_{\mu}$ is DCT of a $1 \mathrm{D}$ vector containing unknown variables, which when solved, will be the object reconstruction for a specific wavelength. Equation 5 is then solved by convex optimisation ${ }^{12}$ algorithm which finds the minimum $\ell_{1}$-norm of the system. Performing an inverse DCT on the output of the solver and reshaping results in solution of the object $O_{\mu}(x, y)$. In summary by employing CGI in a computational system we reduce both the number of physical components and the number of measurements required to form images.

\section{IMAGING APPLICATIONS}

\subsection{Multi-wavelength ghost imaging}

To investigate full color ghost imaging a colored object, in this case a Rubiks cube, was chosen. We take advantage of the four color illumination LEDs (RGB and NIR) for projecting binary patterns, and extract the signals from photodetectors that have been filtered with bandpass colored filters for each wavelength. These signals are used to reconstruct four separate ghost images representing the four color planes of reflected light measured. By overlaying the three R, G and B ghost images we obtain a colored ghost image, similar to typical color imaging methods, as shown in Figure 2. Although we demonstrate only three color ghost imaging using the three visible color LEDs integrated in the projector, this basic technique can be applied to any illumination source which has a suitable reflection response to the DMD device, which may have a wider spectral response of CCD technology offering low cost hyper-spectral imaging within the stated response of the DMD, which is $0.02-0.22 \mu \mathrm{m}$.

Such a high quality reconstruction as shown in Figure 2 requires a large sample size, and since there is a currently a limit on pattern display rate at around $20 \mathrm{~Hz}$, this leads to very long data acquisition timescales. 


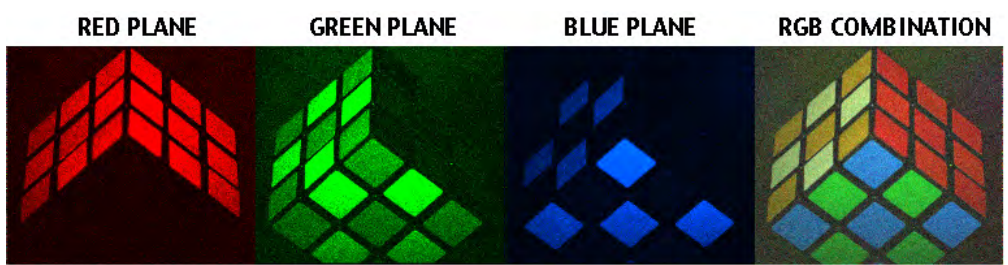

Figure 2. Color plane and RGB combination reconstructions from filtered diode voltage signals under white light illumination at $192 \times 168$ pixel resolution. This is a non-compressed iterative result produced from a very large number of measurements $(>10 \times$ Nyquist).

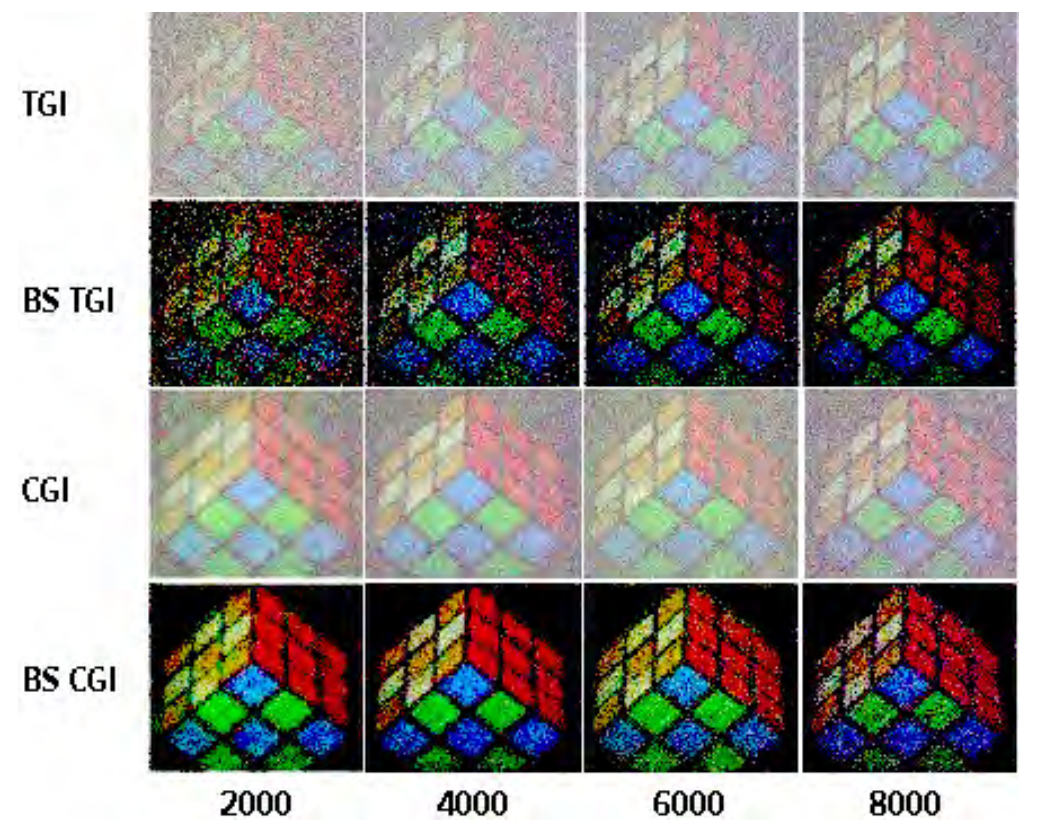

Figure 3. Comparison chart showing reconstructions for increasing sample size by each of the two algorithms employed at $96 \times 84$ pixel resolution. The results of CGI and TGI have been background subtracted and contrast adjusted in the rows donated with BS TGI and BS CGI respectively.

Compressed sensing methods were applied to a smaller sample of the large dataset to attempt faithful image reconstruction in the Sub-Nyquist range. The compressed sensing method shown in Figure 3 highlights the improvements in the overall contrast and clarity of the images compared to the iterative GI algorithm for the same sample size. TGI is still produced for each image reconstruction for a 'live' preview, since it can be implemented with extremely low computational load.

\subsection{Fluorescence ghost imaging}

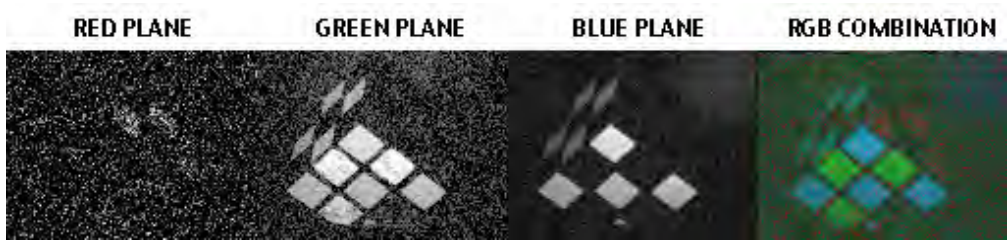

Figure 4. Color plane and RGB combination reconstructions from filtered diode voltage signals under short-wavelength illumination at $128 \times 96$ pixel resolution. Produced from a high measurement number $(>10 \times$ Nyquist $)$ this non-compressed result shows fluorescence in the green plane and fainter fluorescence in the red, blue is simply the reflection plane.

Fluorescence is the emission of photons by certain minerals, dyes and paints when high energy incident light is absorbed and then re-emitted at a longer wavelength and lower energy. This well known phenomenon is 
used extensively for a multitude of purposes including industrial testing techniques and color enhancement in microscopy of biological samples. Normally visible light fluorescence, most commonly produced by ultraviolet stimulation, can be easily observed using CCD cameras. However a Bayer, or otherwise, color filtered commercially available CCD camera can have poor color discrimination and mainly functions in the visible spectrum. Thus a GI approach may be able to offer some distinct advantages over traditional imaging methods.

Fluorescence GI is achieved by careful selection of emission wavelength of the projector for a filtered detector to receive stimulated emission in its specific bandwidth, which has been shown in a classical (non-computational) GI setup. ${ }^{13}$ In order to demonstrate fluorescence GI in our computational compressive projector-based system, certain parts (colored squares on the Rubiks cube) of our test object have been painted with two different fluorescent inks: red and green. We use the same experimental setup as previously described, however the projector is in a single illumination mode, such that it projects monochromatic binary patterns. It is possible to produce true color images of the reflecting and emitting components of the test object, this allows for inherent separation of these two components or combination thereof. In the various color planes of Figure 4 we observe both reflection and fluorescence spectral components of the object, which to the best of our knowledge is the first time this has been shown in such a system.

\subsection{Non-visible ghost imaging}

The true purpose of constructing such a ghost imaging system as that shown in Figure 1 is to take advantage of the broadband response to illumination of the DMD micro-mirror device in order to produce a broad spectrum or selective wavelength image reconstruction, such as hyper-spectral or absorption line imaging. The DLP comes with a fourth, non visible near infrared $(850 \mathrm{~nm})$ LED, so this frequency was selected for convenience to demonstrate the proof of principle result that this GI system can image beyond the visible light spectrum . Using the same technique to obtain fluorescence images, the projector was placed in a single illumination mode and a single filtered photodetector was used to measure the reflected light from each projected pattern at this near-infrared wavelength.

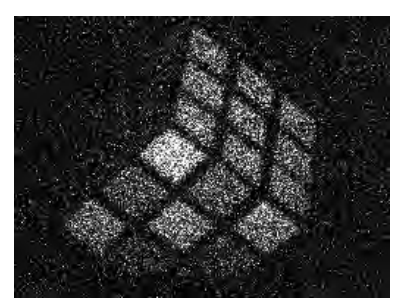

Figure 5. Non-Visible Reconstruction form single filtered diode voltage signals under short-wavelength illumination at $256 \times 192$ pixel resolution. Produced from high measurement number $(5 \times$ Nyquist $)$ this non-compressed result shows a reconstruction of a non-visible wavelength color plane.

Although Figure 5 is only a single non-visible result, the ability to image in a region for which the DMD has an acceptable response to illumination allows for a potentially low cost alternative to current non-visible imaging devices which have the inherent difficulty in producing high quality pixellated arrays for such frequencies.

\section{CONCLUSIONS}

In conclusion we have shown that GI systems can be adapted to enable full-color, multi-wavelength and multicomponent fluorescent images of real objects. The use of light projectors in such imaging devices shows promise as being a practical, low cost and efficient device for multi-spectral imaging applications, particularly at frequencies of the electromagnetic spectrum where CCD imaging technology may be unsuitable. The use of single pixel detectors may offer low data requirements to transfer images, provided the patterns used to generate them are known. Although the pattern display rate of our system averaged $20 \mathrm{~Hz}$, in principle this particular projector technology can operate up to $5 \mathrm{kHz}$. With further development this system could become a useful multi-wavelength/hyper-spectral camera with additional benefits in fluorescence imaging. 


\section{REFERENCES}

1. Pittman, T. B., Shih, Y. H., Strekalov, D. V., and Sergienko, A. V., "Optical imaging by means of twophoton quantum entanglement," Phys. Rev. A 52, R3429-R3432 (Nov 1995).

2. Bennink, R. S., Bentley, S. J., and Boyd, R. W., "“two-photon" coincidence imaging with a classical source," Phys. Rev. Lett. 89, 113601 (Aug 2002).

3. Gatti, A., Brambilla, E., Bache, M., and Lugiato, L. A., "Ghost imaging with thermal light: Comparing entanglement and classicalcorrelation," Phys. Rev. Lett. 93, 093602 (Aug 2004).

4. Gatti, A., Brambilla, E., Bache, M., and Lugiato, L. A., "Correlated imaging, quantum and classical," Phys. Rev. A 70, 013802 (Jul 2004).

5. Ferri, F., Magatti, D., Gatti, A., Bache, M., Brambilla, E., and Lugiato, L. A., "High-resolution ghost image and ghost diffraction experiments with thermal light," Phys. Rev. Lett. 94, 183602 (May 2005).

6. Shapiro, J. H., "Computational ghost imaging," Phys. Rev. A 78, 061802 (Dec 2008).

7. Erkmen, B. I. and Shapiro, J. H., "Ghost imaging: from quantum to classical to computational," Adv. Opt. Photon. 2, 405-450 (Dec 2010).

8. Ferri, F., Magatti, D., Lugiato, L. A., and Gatti, A., "Differential ghost imaging," Phys. Rev. Lett. 104, 253603 (Jun 2010).

9. Sun, B., Welsh, S. S., Edgar, M. P., Shapiro, J. H., and Padgett, M. J., "Normalized ghost imaging," Opt. Express 20, 16892-16901 (Jul 2012).

10. Katz, O., Bromberg, Y., and Silberberg, Y., "Compressive ghost imaging," Applied Physics Letters 95(13), 131110 (2009).

11. Donoho, D., "Compressed sensing," Information Theory, IEEE Transactions on 52, 1289-1306 (april 2006).

12. Candes, E. and Recht, B., "Exact matrix completion via convex optimization," Foundations of Computational Mathematics 9, 717-772 (2009). 10.1007/s10208-009-9045-5.

13. Tian, N., Guo, Q., Wang, A., Xu, D., and Fu, L., "Fluorescence ghost imaging with pseudothermal light," Opt. Lett. 36, 3302-3304 (Aug 2011). 